In Situ

Revue des patrimoines

\section{In Situ}

Revue des patrimoines

27 | 2015

Le cheval et ses patrimoines (2e partie)

\title{
Le nouveau musée national des Carrosses, un défi !
}

\author{
Silvana Bessone
}

\section{OpenEdition}

Journals

Édition électronique

URL : http://journals.openedition.org/insitu/11941

DOI : 10.4000/insitu. 11941

ISSN : 1630-7305

\section{Éditeur}

Ministère de la culture

\section{Référence électronique}

Silvana Bessone, « Le nouveau musée national des Carrosses, un défı ! », In Situ [En ligne], 27 | 2015, mis en ligne le 02 novembre 2015, consulté le 03 mai 2019. URL : http://journals.openedition.org/ insitu/11941 ; DOl : 10.4000/insitu.11941

Ce document a été généré automatiquement le 3 mai 2019.

\section{(c) (i) $\odot$}

In Situ Revues des patrimoines est mis à disposition selon les termes de la licence Creative Commons Attribution - Pas d'Utilisation Commerciale - Pas de Modification 4.0 International. 


\title{
Le nouveau musée national des Carrosses, un défi!
}

\author{
Silvana Bessone
}

1 La décision de faire bâtir un édifice pour installer le nouveau musée des Carrosses (Museu dos Coches Reaes), dans un terrain en face de l'actuel musée, à Belém, prise par le gouvernement portugais le 30 avril 2008 n'a pas été, comme on le pourrait croire à première vue, une idée passagère mais bien au contraire le résultat d'un long processus initié il y a plus d'un siècle.

Figure 1

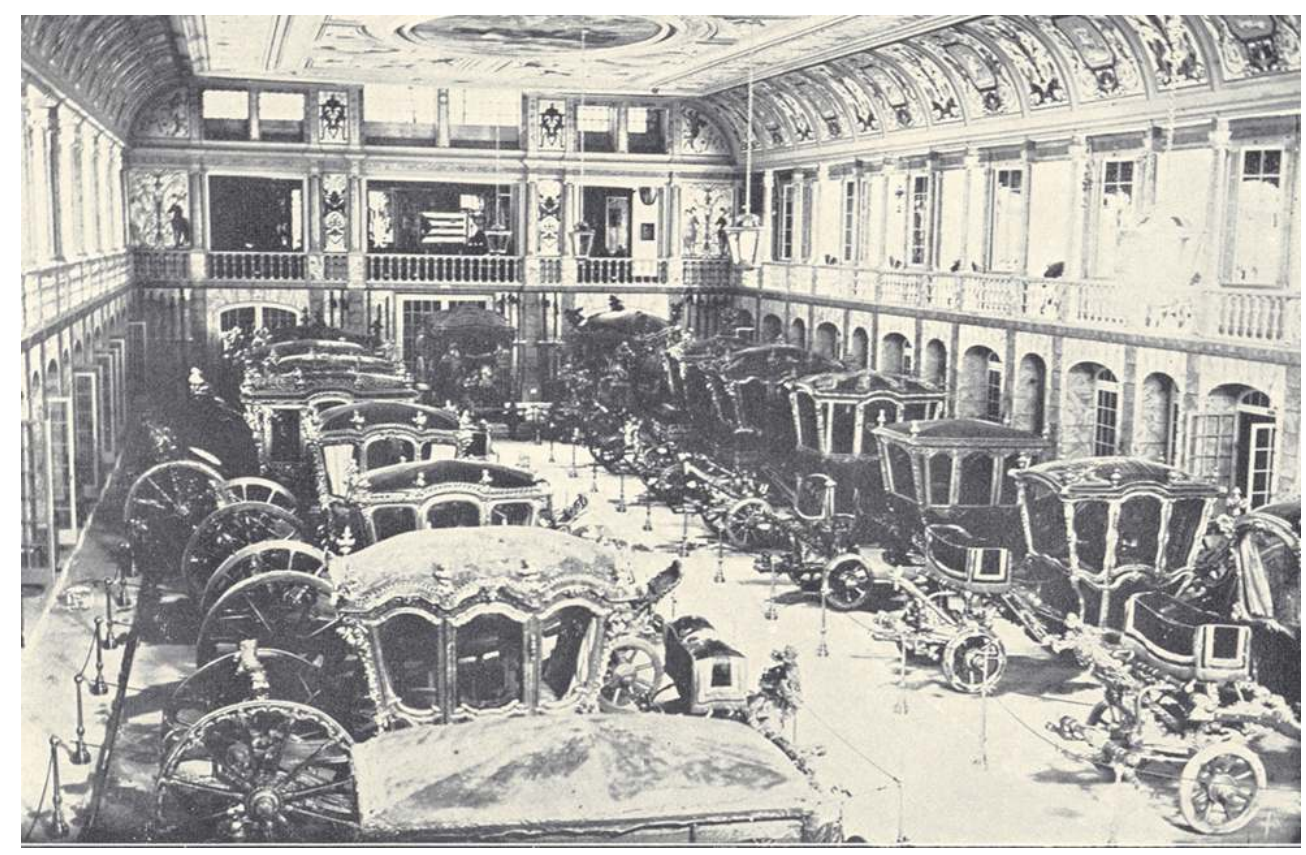

Musée des Carrosses royaux, 1905.

Phot. Bessone, Silvana, Beltrão, Pedro. ( $)$ Archives du musée national des Carrosses (MNC). 
En fait, le musée des Carrosses royaux inauguré le 23 mai 1905 a fait face, dès sa naissance, à un problème de manque d'espace (fig. 1), décelé immédiatement par sa fondatrice, la reine Amélie d'Orléans qui, en 1906, un an après l'inauguration, commanda à Rosendo Carvalheira, architecte des Palais royaux, un nouveau projet pour l'agrandissement du musée vers le nord, dans la cour des Dames, de façon à accueillir et présenter dans de meilleures conditions la collection de voitures de la Maison royale portugaise (fig. 2).

Figure 2

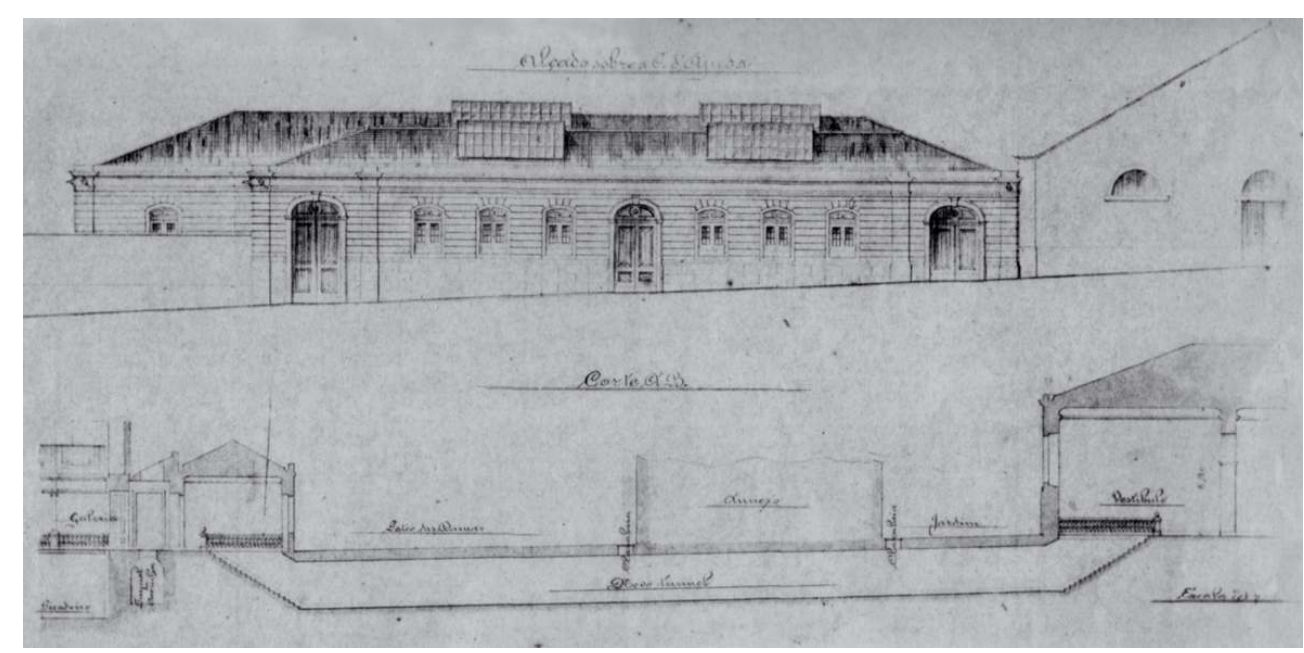

Projet pour l'agrandissement du musée, 1906. Passage souterrain.

Phot. Bessone, Silvana, Beltrão, Pedro. (c) Archives du MNC.

Le projet prévoyait la construction d'un nouveau bâtiment relié à l'ancien manège par un passage souterrain et une entrée latérale vers la chaussée d'Ajuda.

4 L'assassinat du roi Charles $I^{\text {er }}$, survenu le $1^{\text {er }}$ février 1908, empêcha la réalisation de ce projet, et le manque d'espace dans le musée devint une préoccupation constante pour ses responsables qui par divers moyens, essayèrent de résoudre le problème.

Après l'instauration de la République, le 5 octobre 1910, le musée des Carrosses royaux changea non seulement changé de nom mais devint le premier musée national du Portugal, sous l'appellation, dès le 26 mai 1911, de « musée national des Carrosses ».

6 Avec l'intégration de nouvelles voitures provenant de différents dépôts, des palais royaux ou de l'Église, le gouvernement commanda en 1941 un nouvel espace d'exposition à l'architecte Raul Lino, construit du côté est de l'édifice, tirant parti du terrain d'un ancien potager (fig. 3). La construction respectait la trace de l'édifice, reproduisant dans la façade orientale les éléments de la façade principale de l'ancien bâtiment tout en les adaptant aux contraintes du terrain en pente. 
Figure 3

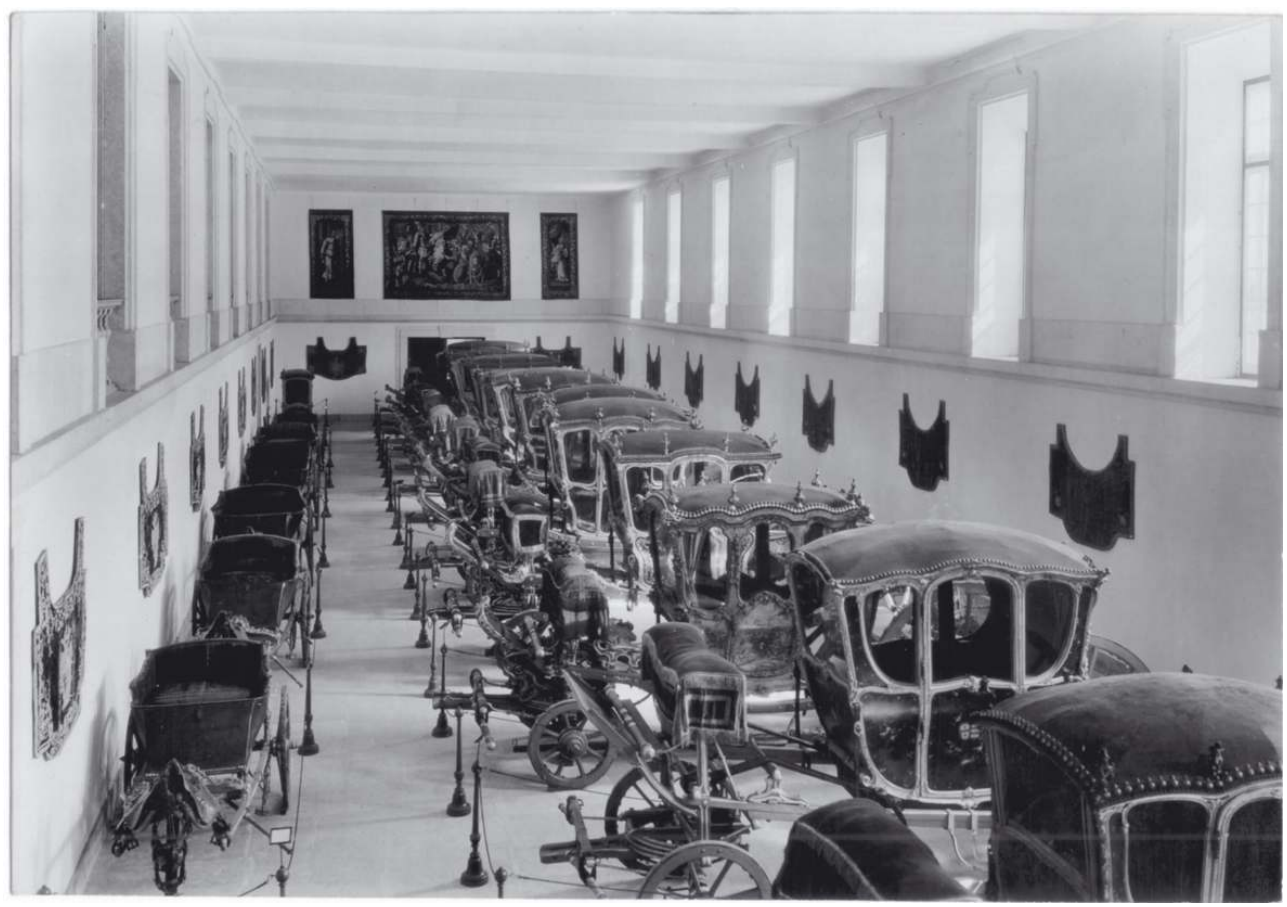

Nouvelle salle d'exposition, 1944.

Phot. Bessone, Silvana, Beltrão, Pedro. (c) Archives du MNC

7 Malgré la construction de ce nouvel espace, inauguré en 1944, le musée manquait encore de place pour présenter sa collection et accueillir convenablement le public. Avec le développement du tourisme, le musée faisait face à une augmentation constante des visiteurs, ce qui entraînait des conditions de visite encore plus difficiles. Par ailleurs, le service éducatif se développait et exigeait de nouveaux espaces de travail, les réserves insuffisantes ne correspondaient plus aux exigences actuelles de conservation, la boutique du musée ne répondait plus aux demandes des visiteurs; tous ces problèmes constituaient assez de motifs pour que l'on recherche une solution adaptée au musée portugais le plus visité (fig. 4). 
Figure 4

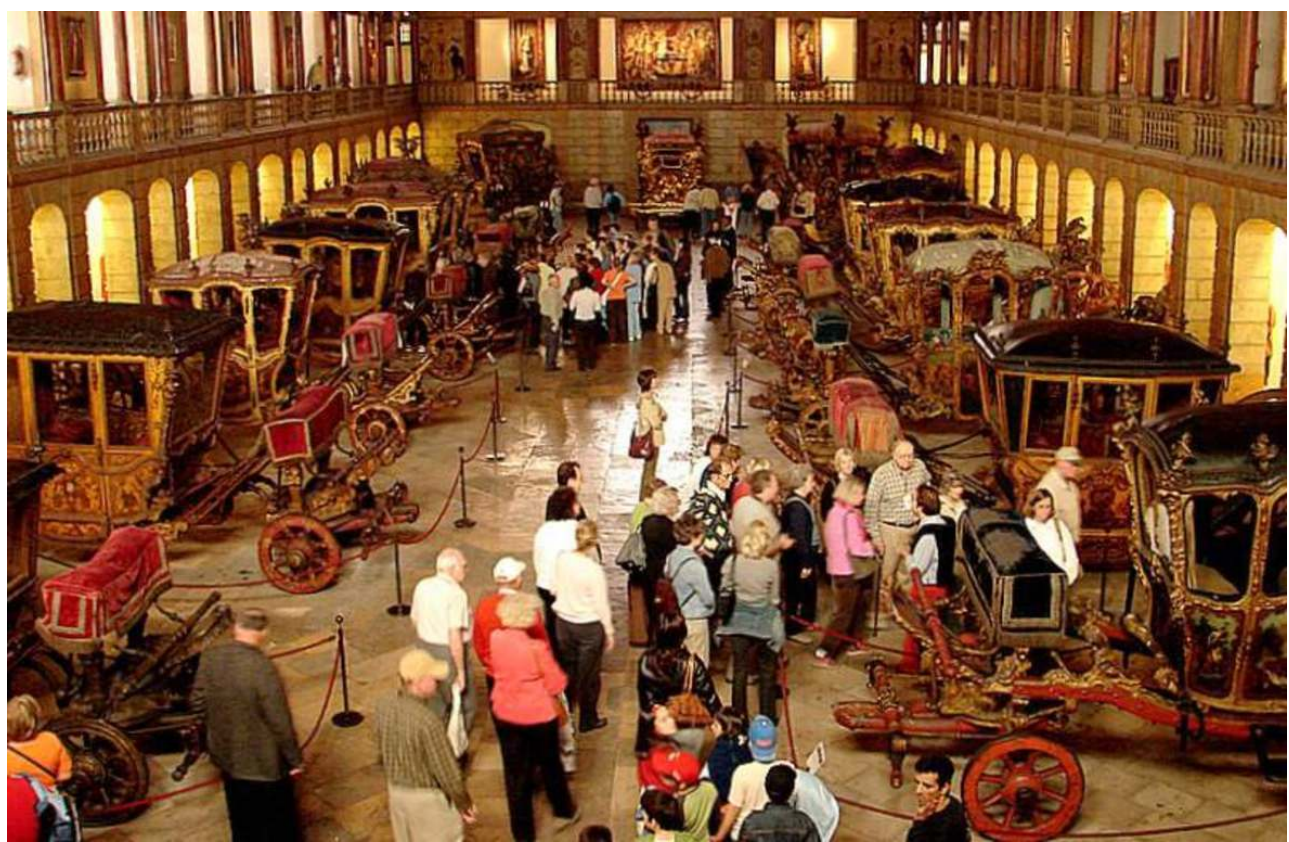

Les visiteurs au musée national des Carrosses, 1995.

Phot. Bessone, Silvana, Beltrão, Pedro. (c) Archives du MNC.

Finalement, en 1995, une possibilité se présenta après la désaffectation d'un bâtiment militaire occupé par les usines générales de l'Armée, sur un terrain en face du musée. J'ai moi-même proposé tout de suite au secrétaire d'État à la Culture de l'époque d'acheter ce terrain pour y installer un nouvel édifice complémentaire qui pourrait agrandir le musée, à l'instar de plusieurs musées étrangers qui ont relié l'ancien édifice du musée avec une construction nouvelle.

9 Le terrain de $17000 \mathrm{~m}^{2}$ a été aussitôt acheté mais il a fallu attendre jusqu'à 2008 pour que le gouvernement portugais décide de faire construire un nouveau musée (fig. 5). Une fois prise la décision politique, c'est à nous que revenait la responsabilité d'élaborer le programme muséologique pour le nouveau musée, avec une préoccupation naturelle quant au développement de son projet. 


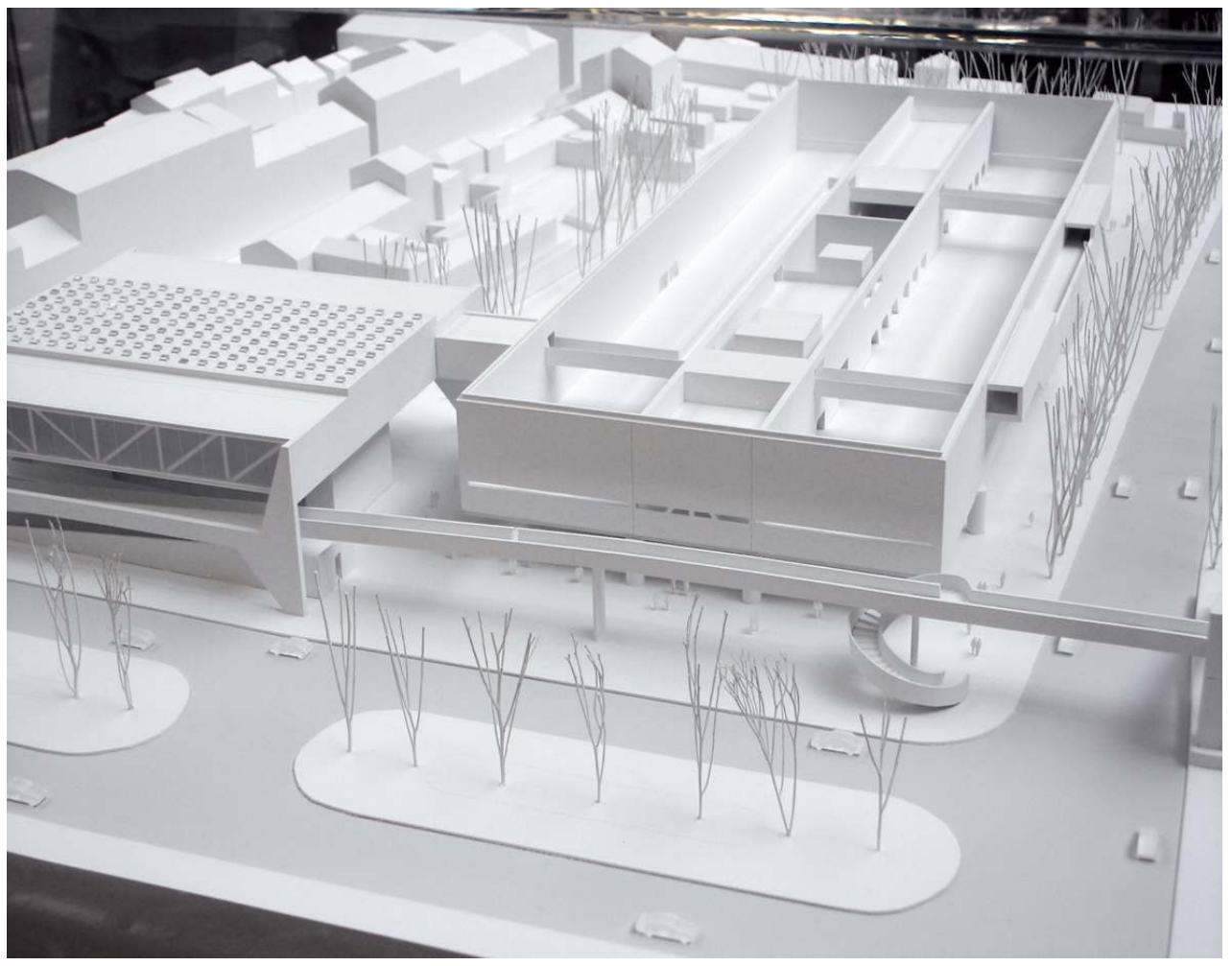

Maquette du nouveau musée, 2008.

Phot. Bessone, Silvana, Beltrão, Pedro. (c) Archives du MNC

Deux objectifs marquèrent dans l'immédiat l'élaboration de ce programme. En premier lieu, démontrer aux responsables politiques la nécessité de conserver dans les installations d'origine la mémoire de ce musée centenaire, véritable icône de la ville de Lisbonne et premier musée du Transport dans le monde.

nous avons proposé que dans le musée actuel, une exposition consacrée au cheval et à l'art équestre et un groupe de dix voitures subsiste, permettant au public de compléter la visite du nouveau musée en revisitant l'ancien, et laissant aussi la possibilité d'utiliser cet espace pour organiser diverses manifestations dans lesquelles le musée national des Carrosses a joué un rôle pionnier au Portugal depuis plus de vingt-cinq ans.

En deuxième lieu, doter le nouvel édifice de tous les équipements nécessaires, non seulement au plein accomplissement de sa mission en tant que lieu de recherche, conservation et valorisation de ses collections, mais aussi en assumant la fonction du musée en tant que générateur de développement économique.

Dans ce domaine, le programme muséologique prend en compte une nouvelle dimension dans le domaine de la conservation et de la restauration des voitures hippomobiles, avec la création d'une école destinée à préparer des conservateurs-restaurateurs spécialisés dans ce type de patrimoine; à cette fin, le prestige du musée national des Carrosses a motivé une recherche permanente de soutiens auprès de collectionneurs internationaux et même de quelques institutions muséales.

Le nouveau musée a un espace pour le développement et le perfectionnement des techniques de traitement, de conservation préventive et de restauration, en collaboration 
avec quelques activités artisanales liées à la construction de voitures hippomobiles telles que la sellerie, la bourrellerie et la passementerie, métiers qui se trouvent actuellement en voie d'extinction et dont il est capital de recueillir les savoir-faire.

La zone destinée à la conservation et au traitement des voitures et autres objets comprend en plus de la salle de travail une salle pour désinsectiser les objets par anoxie dynamique et un compartiment pour des produits chimiques.

De la même façon, l'installation d'un considérable espace pour les réserves, d'environ $1000 \mathrm{~m}^{2}$, convenablement compartimentées et équipées d'une climatisation adaptée à la conservation des différentes typologies d'objets, offre de meilleures conditions de travail et la possibilité de rassembler quelques objets encore dispersés.

Mais dans le défi que présente ce nouveau musée, la captation de nouveaux publics et la nécessité de satisfaire le plus grand nombre de visiteurs ont également été prises en compte dans le programme muséologique (fig. 6).

Figure 6

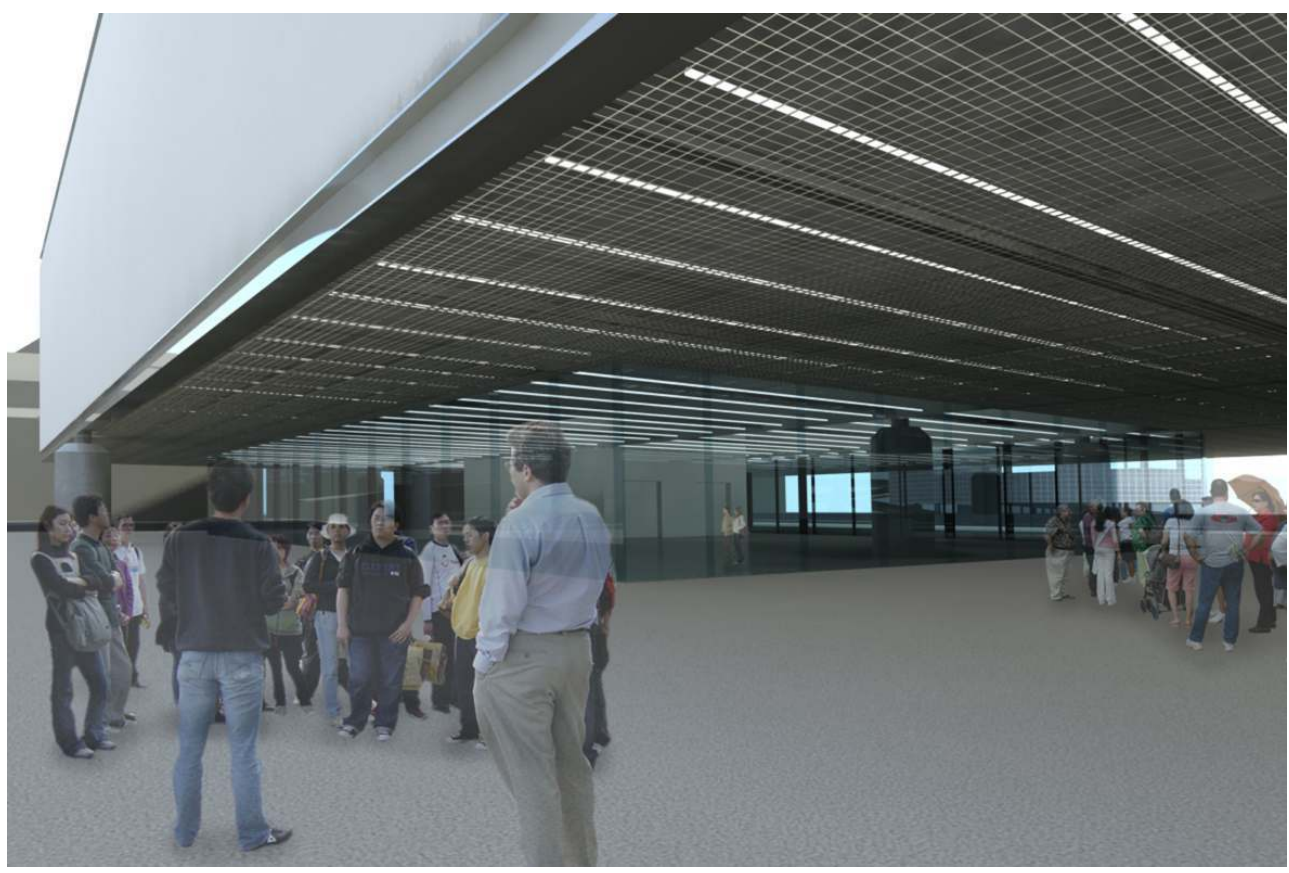

Image virtuelle de l'entrée du nouveau musée. Présentation du projet d'architecture, 2008. Architecte Paulo Mendes da Rocha.

Phot. Bessone, Silvana, Beltrão, Pedro. (c) Archives du MNC.

De nouveaux équipements ont été conçus pour accueillir le public, comprenant une cafétéria, un restaurant et deux emplacements commerciaux pour les boutiques du musée. À part cela, l'espace pour les activités du service éducatif, la bibliothèque et les archives a été agrandi et un auditorium de 350 places a été construit, avec un accès indépendant, pour produire plusieurs initiatives culturelles liées à l'activité du musée ou encore être loué pour d'autres événements (fig. 7). 
Figure 7

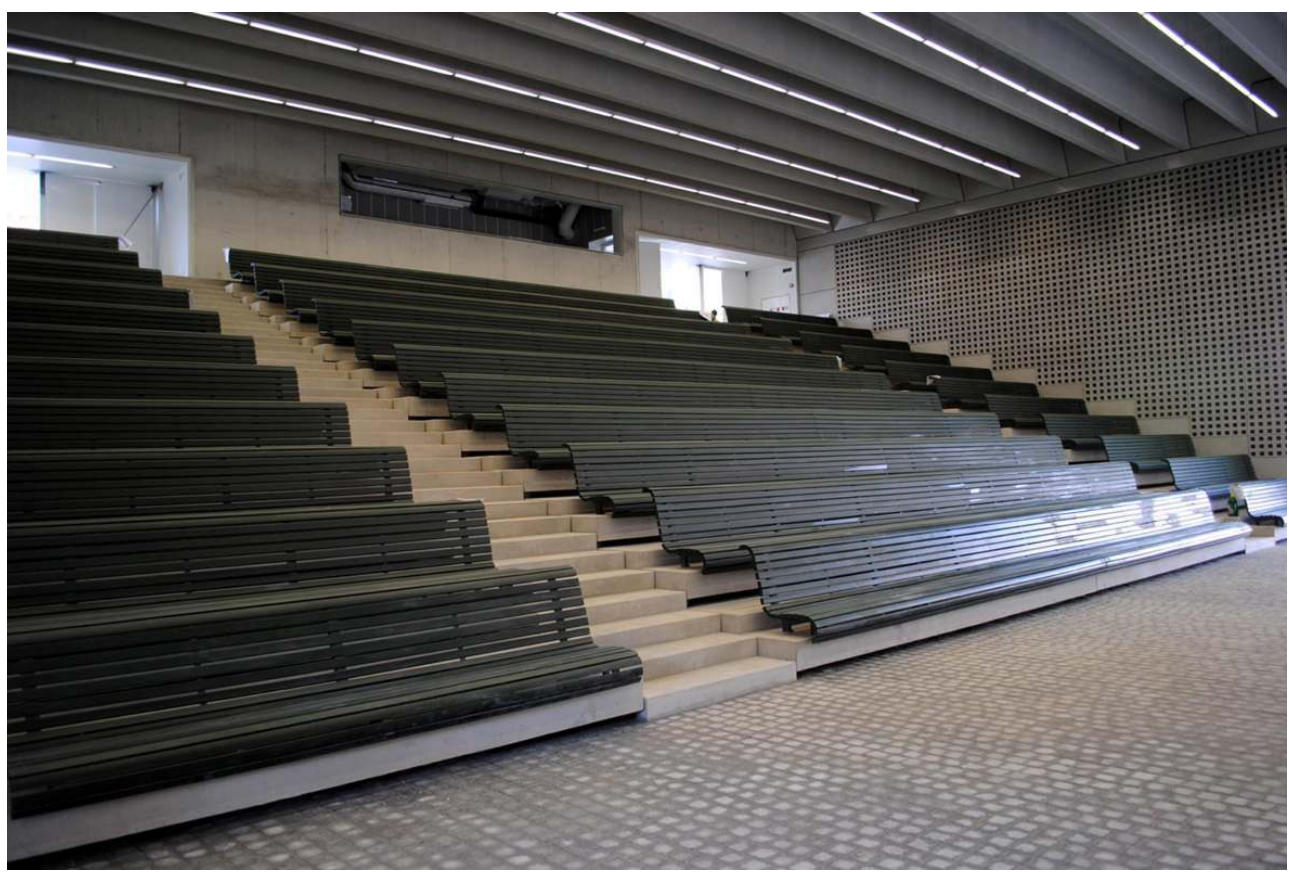

Auditorium du nouveau musée, 2012.

Phot. Bessone, Silvana, Beltrão, Pedro. (c) Archives du MNC

Cependant, malgré l'importance muséologique accordée par le gouvernement au nouveau musée national des Carrosses, le ministère de la Culture et particulièrement l'Institut des Musées et de la Conservation, qui exerce la tutelle des musées d'État, ont été tenus un peu à l'écart dans la conduite du programme approuvé. Pour cette raison, le début de l'opération a été difficile. Et la participation des conservateurs du musée, dans l'équipe multidisciplinaire qui élaborait le projet architectural, bien qu'établie dans la théorie muséologique, s'est résumée à une série de réunions dans lesquelles les solutions du projet étaient présentées déjà arrêtées et protégées par la figure du «concept architectonique " défendue par l'équipe et sans qu'il y ait moyen de les modifier. Finalement, en dépit de plusieurs batailles perdues, et d'autres gagnées, le nouveau musée représente une plus-value significative, que ce soit pour la présentation des collections ou pour la commodité des visiteurs (fig. 8). 


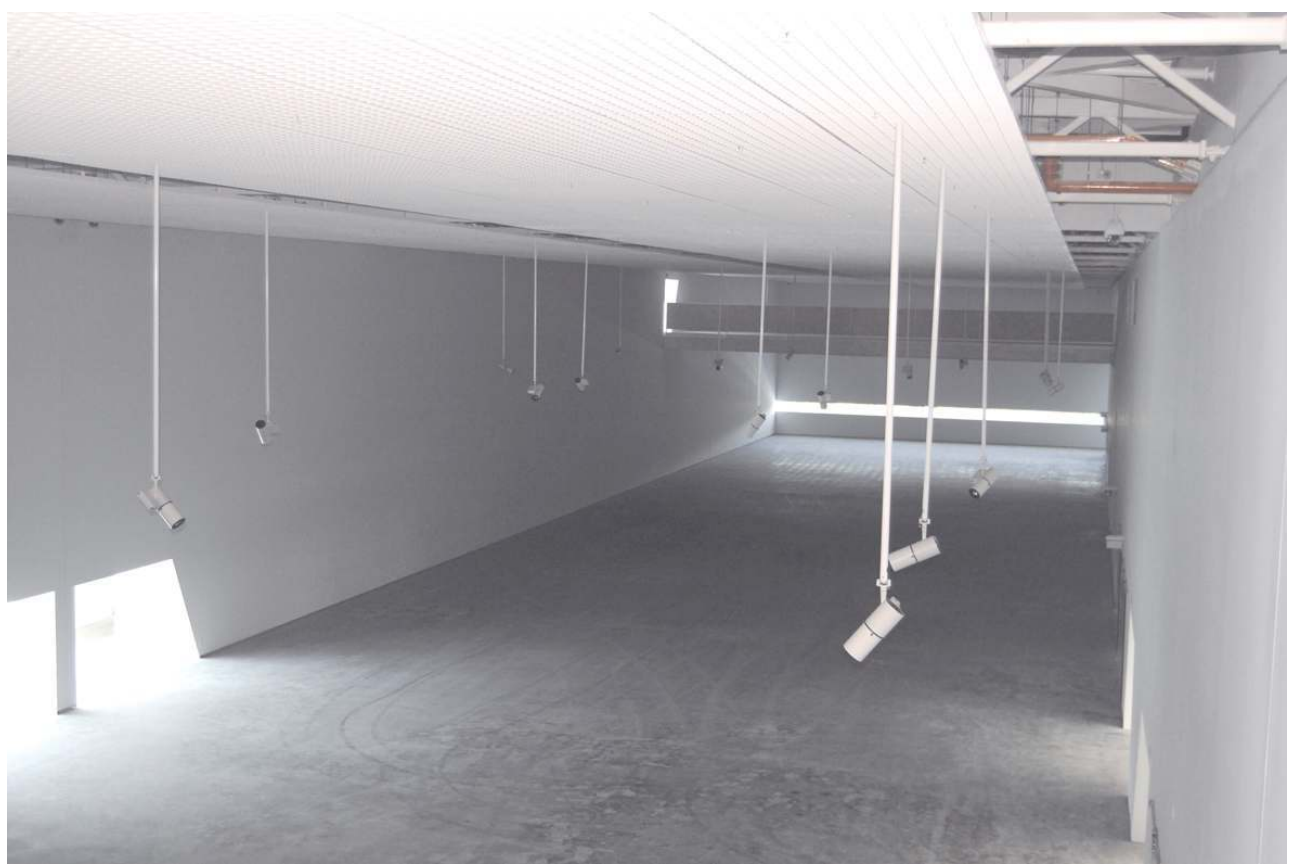

Galerie d'exposition. Vue de l'aile sud, 2012.

Phot. Bessone, Silvana, Beltrão, Pedro. (c) Archives du MNC. dans le nouvel édifice, avec une surface d'exposition d'environ $11000 \mathrm{~m}^{2}$, permet au public d'effectuer un parcours à travers l'évolution du transport hippomobile utilisé par l'aristocratie et le clergé de la fin $\mathrm{du} \mathrm{xvI}^{\mathrm{e}}$ au début $\mathrm{du} \mathrm{xx}^{\mathrm{e}}$ siècle, en observant les changements stylistiques ou les évolutions techniques qui nous ont conduits à l'automobile.

21 Cet aspect représente sans doute un avantage indiscutable, non seulement pour la recherche mais également pour l'enrichissement de l'exposition et la présentation au public, favorisant une plus grande reconnaissance internationale de la valeur de cette fameuse collection de voitures hippomobiles utilisées par l'aristocratie européenne. croissant de visiteurs. Le musée national des Carrosses installé dans un édifice contemporain dessiné par l'architecte brésilien Paulo Mendes da Rocha représente un nouveau pôle d'attraction que l'exceptionnelle collection justifie et constitue une marque de modernité culturelle pour le pays, en étant par ailleurs situé dans un quartier privilégié de la ville de Lisbonne.

exposition est organisée en plusieurs noyaux, suivant une séquence chronologique mais aussi regroupés autour de thématiques comme le baroque italien, le baroque portugais, le carrosse et la berline, le transport des cardinaux, les promenades en plein air, les voitures de chasse, les voitures pour enfants, le transport sans roues et le transport collectif. L'exposition se termine par la présentation d'un cortège avec différents types de voitures $\mathrm{du} \mathrm{XIX}^{\mathrm{e}}$ siècle.

Les solutions muséographiques intégrées dans le parcours de l'exposition recourent aux nouvelles technologies pour mettre à la disposition des visiteurs des bornes 
informatiques où ils peuvent obtenir en plusieurs langues des renseignements sur les voitures exposées (fig. 9). Des éléments sur l'origine, le constructeur, le propriétaire, le décor et l'histoire de son utilisation, accompagnée de la projection d'une série de documents iconographiques illustrant son usage, complètent la compréhension de l'évolution technique et stylistique mise en œuvre dans la construction des voitures au fil des siècles et aussi, celui du rôle croissant que leur utilisation a pris dans la représentation du pouvoir et du statut social de leurs propriétaires.

\section{Figure 9}

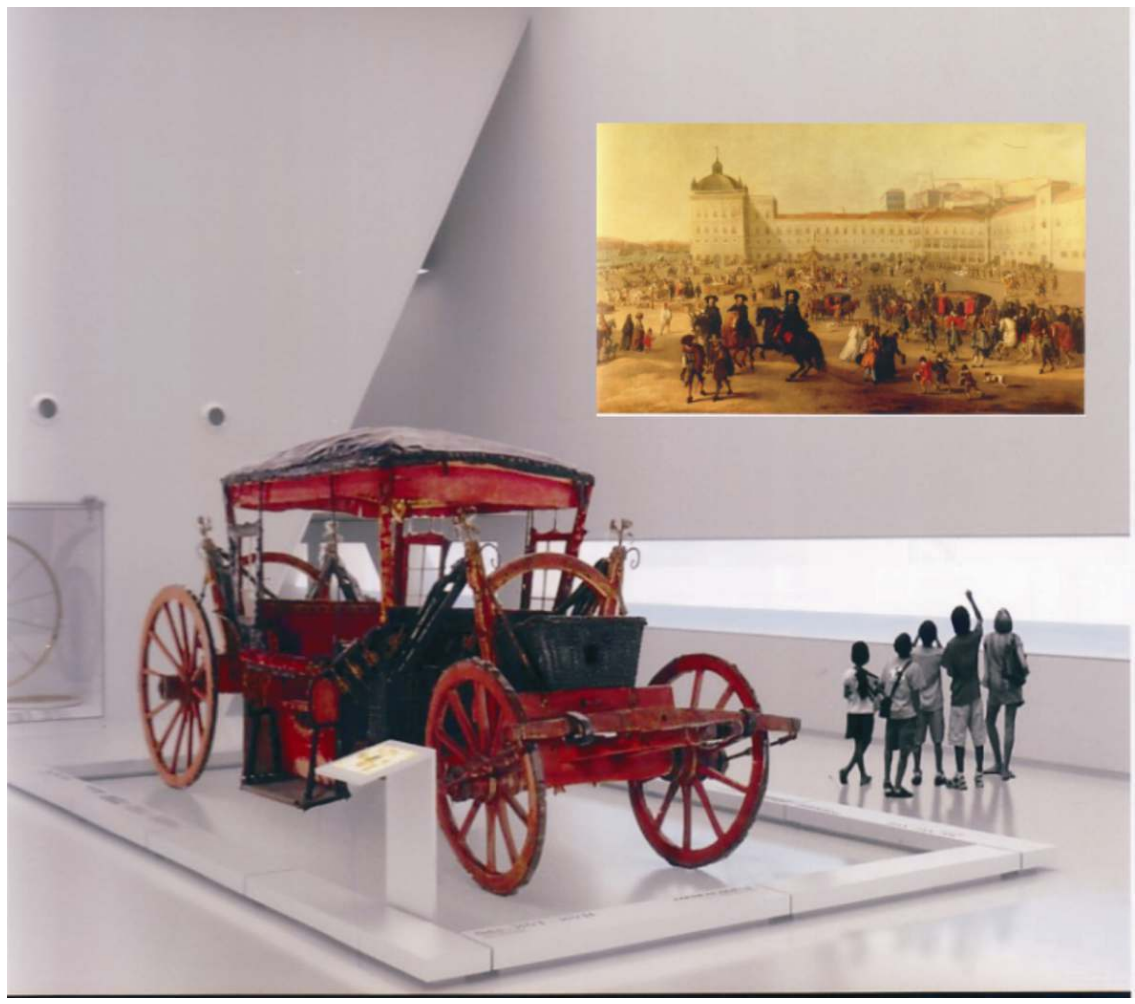

Image virtuelle du projet muséographique, 2009.

Phot. Bessone, Silvana, Beltrão, Pedro. ( ) Archives du MNC

Bénéficier d'un nouvel édifice a permis de présenter une « lecture intégrale » des voitures et de définir l'existence de deux parcours avec une durée de visite différenciée (fig. 10). 


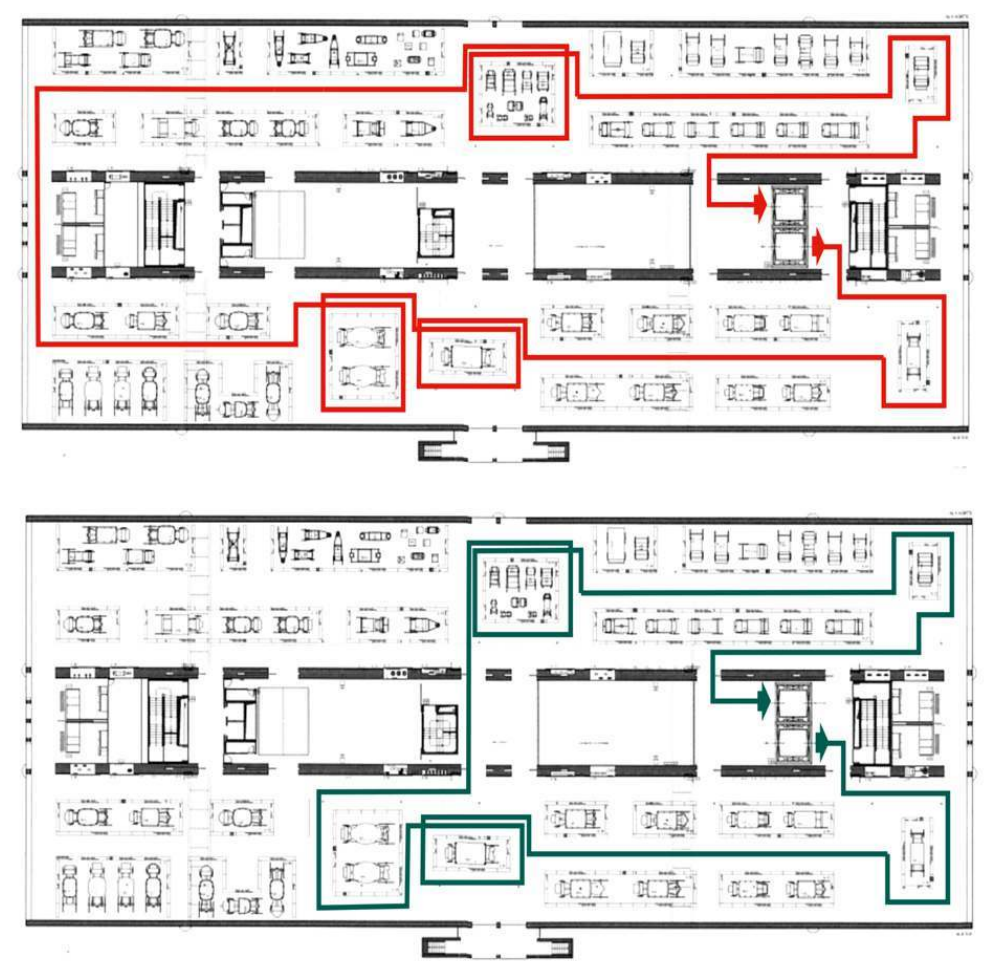

Les parcours de visite. Programme muséologique.

Phot. Bessone, Silvana, Beltrão, Pedro. (c) Archives du MNC

On sert mieux les besoins des opérateurs touristiques pour les groupes avec des temps de visite limités, assurant dans tous les cas aux visiteurs un aperçu des voitures les plus importantes.

Pour l'exposition des petits objets, la solution retenue par les architectes a été de faire construire des vitrines intégrées dans la structure métallique que forme l'édifice, afin de faciliter la circulation du public et des voitures (fig. 11). 
Figure 11

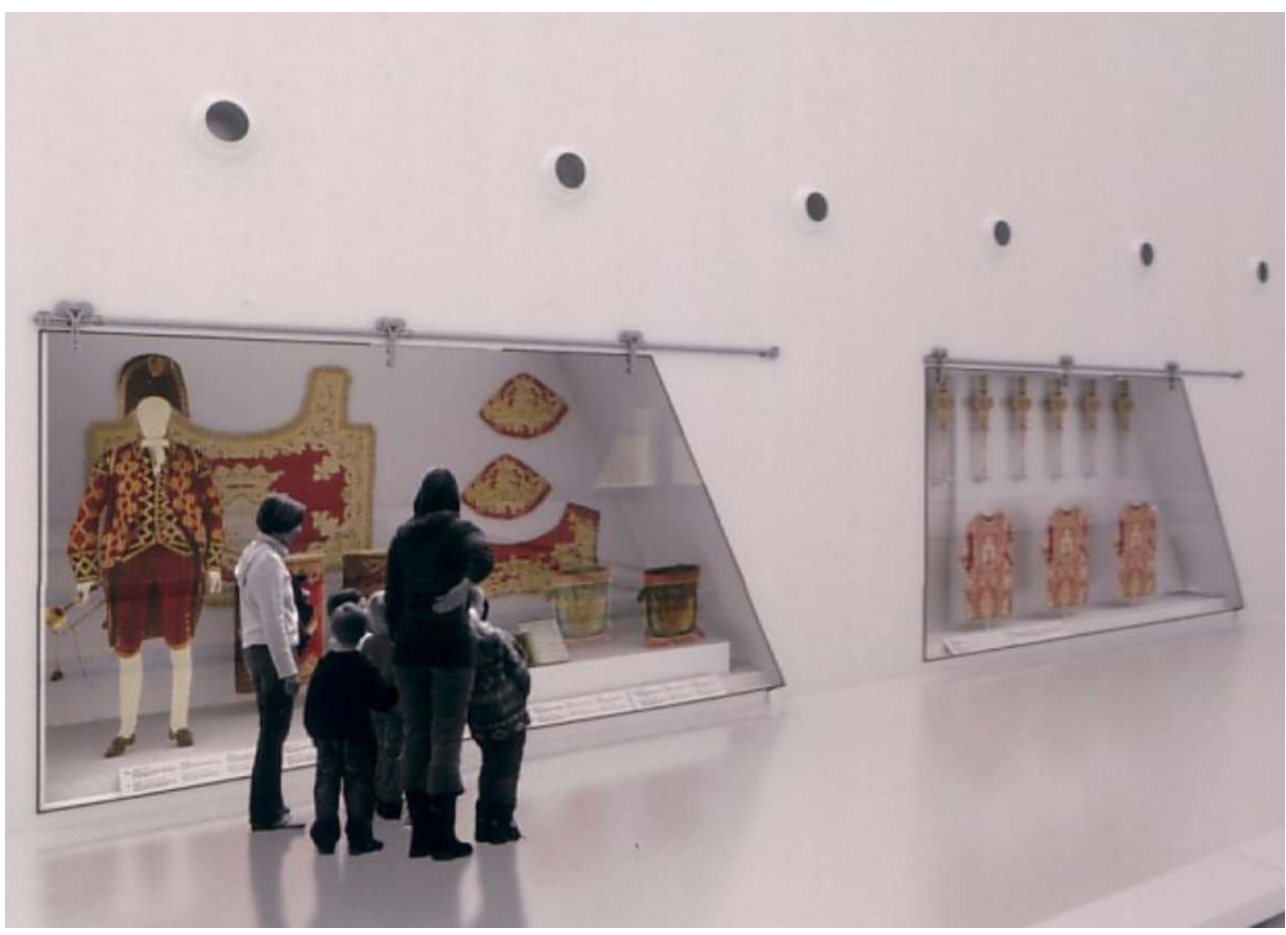

Image virtuelle du projet muséographique, 2009.

Phot. Bessone, Silvana, Beltrão, Pedro. ( $)$ Archives du MNC.

Plus d'un siècle après la commande par Amélie d'Orléans d'un projet pour agrandir le musée des Carrosses royaux, par manque d'espace, le nouveau musée national des Carrosses pourra finalement accueillir convenablement sa magnifique collection (fig. 12). 
Figure 12

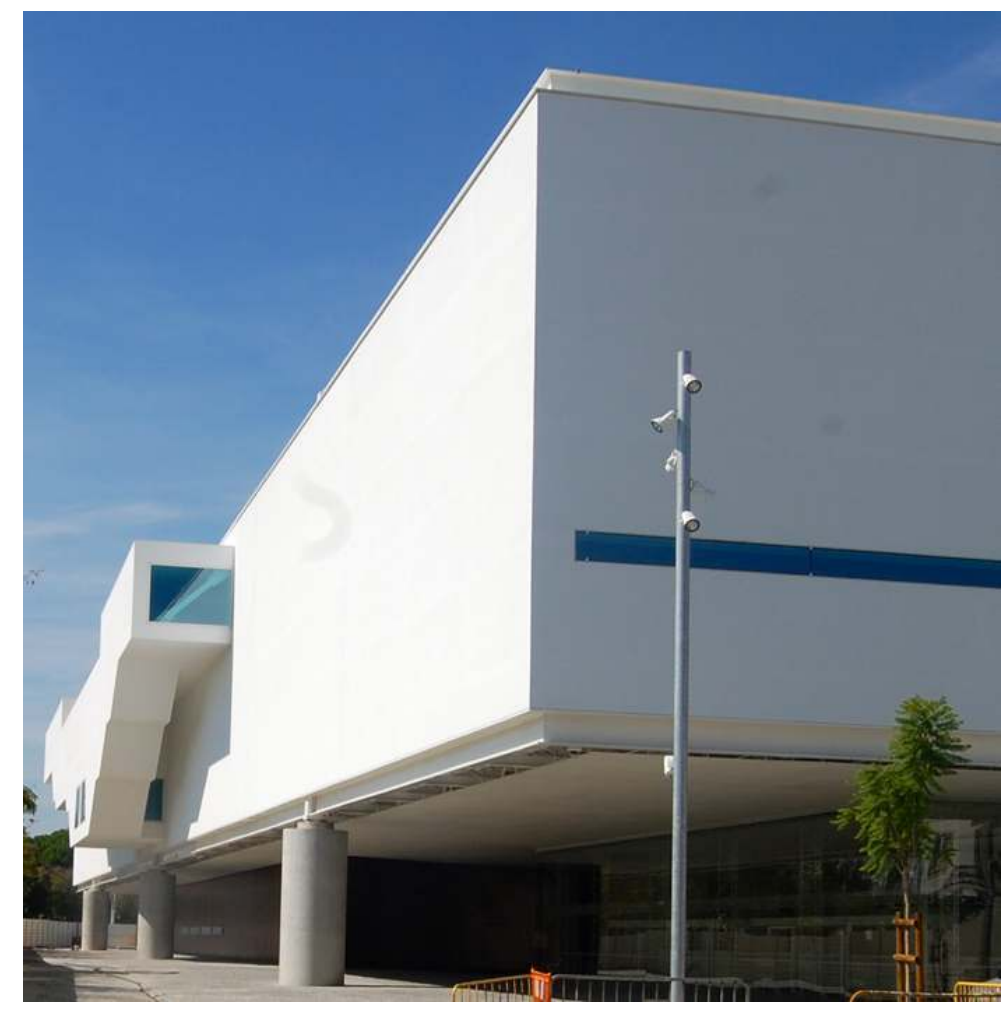

Le nouveau musée des Carrosses. Façade sud, 2012.

Phot. Bessone, Silvana, Beltrão, Pedro. (c) Archives du MNC.

\section{RÉSUMÉS}

Après plus de cent ans d'existence dans ses installations d'origine, résultant d'une parfaite transformation de l'ancien manège royal de Belém pour exposer les voitures hippomobiles appartenant à la Maison royale de Portugal, le gouvernement portugais a décidé la construction d'un nouvel édifice pour accueillir convenablement une des plus importantes collections de voitures hippomobiles allant de la fin $\mathrm{du} \mathrm{XVI}^{\mathrm{e}}$ au début du $\mathrm{XX}^{\mathrm{e}}$ siècle. Cette décision s'est révélé un énorme défi pour la muséologie portugaise.

The new national carriage museum, a challenge! The horse-drawn vehicles belonging to the Portuguese royal family were displayed for over a century in their original location, the former royal riding school at Belém, perfectly adapted to this museum use. But the government has recently decided to have a new museum building erected, worthy of this extremely rich collection of vehicles, dating from the end of the sixteenth century to the beginning of the twentieth. This decision represents a huge challenge for the country's museology specialists. 
INDEX

Mots-clés : décision, programme, muséologie, projet, nouveau musée, carrosses, défi

Keywords : museology, project, new museum, challenge

\section{AUTEUR}

\section{SILVANA BESSONE}

Museu Nacional dos Coches, directrice sbessone@sapo.pt 